\title{
EFFECT OF TEMPERATURE ON ANISOTROPY IN FORMING SIMULATION OF ALUMINUM ALLOYS
}

\author{
S. Kurukuri ${ }^{1,2 *}$, A. Miroux ${ }^{1,3}$, M. Ghosh ${ }^{1,3}$, A.H. van den Boogaard ${ }^{2}$ \\ ${ }^{1}$ Materials Innovation Institute, The Netherlands \\ ${ }^{2}$ University of Twente, Faculty of Engineering Technology, The Netherlands \\ ${ }^{3}$ Delft University of Technology, Faculty of $3 \mathrm{mE}$, The Netherlands
}

\begin{abstract}
A combined experimental and numerical study of the effect of temperature on anisotropy in warm forming of AA 6016-T4 aluminum was performed. The anisotropy coefficients of the Vegter yield function were calculated from crystal plasticity models with an adequate combination of extra slip systems. Curve fitting was used to fit the anisotropy coefficients calculated at discrete temperatures. This temperature dependent constitutive model was successfully applied to the coupled thermo-mechanical analysis of deep drawing of aluminum sheet and results were compared with experiments.
\end{abstract}

KEYWORDS: warm forming, material anisotropy, yield function, temperature effect

\section{INTRODUCTION}

For the accurate numerical simulation of sheet metal forming, the use of an appropriate material model is of vital importance. Particularly for warm forming simulation, an accurate coupled thermo-mechanical material model for the anisotropic yield function with temperature and rate dependent hardening is required. Use of anisotropic material models in numerical analysis requires thorough material characterization under multiple loading conditions. Since the material anisotropy and hardening behavior change with higher temperatures, the anisotropy coefficients and the hardening behavior must be determined as a function of temperature to perform accurate thermomechanical finite element analysis for these materials. Prior research available for simulation of warm forming processes focuses only on the effect of elevated temperature on the evolution of the flow stress. These include [1-3]. Boogaard and Huétink [3] characterized the behavior of AA5754-O for which two types of flow laws were used: the modified power law and the physically based Bergström model. The Vegter [4] yield surface used in this case was assumed to remain constant with respect to changing temperatures. Only the coefficients of the flow laws were fitted as function of temperature and strain rate. The predictions of the material model, however, underestimated the values of the punch load in both models. Recently, Kurukuri et al. [5] presented the warm deep drawing simulations using the more advanced physically based Nes hardening model for 5754-O alloy and still it underestimates the punch force, even though it performs better than the Bergström model. In all the above works,

*Corresponding author: S. Kurukuri, P.O. Box 217, 7500 AE Enschede, The Netherlands, +31-53-4894567, s.kurukuri@m2i.nl the effect of temperature on the yield locus shape and anisotropy coefficients were not fully explored.

Since, the choice of yield function is essential in material modeling, much effort has been made for experimental observations of yield loci on several types of metals by many researchers. It is well known that the inplane biaxial tension of a cruciform shaped specimen or plane strain tension and simple shear tests are suitable for the investigation of yield locus of sheet metals, but all of these experiments have been performed only at room temperature. Especially in the last decade, very accurate anisotropic yield functions were proposed for sheet metal forming simulations at room temperature, such as the planar anisotropic Vegter yield locus based on experimental measurements and Barlat yield functions for the aluminum alloys.

However, a very limited experimental data of yield loci at elevated temperatures have been reported. This is because the determination of yield loci at elevated temperatures is not an easy task. Very recently, Geiger et al. [6] designed an experimental setup for the biaxial tensile testing of sheet metal at elevated temperatures. In this work, an effort is being made to use the combination of experimental measurements and crystal plasticity model to identify the yield locus parameters at elevated temperature by activating more slip systems.

\section{MATERIAL MODEL}

Material models for plastic deformation that are used in process simulations commonly apply a separation of the model in a yield surface and an evolution of the yield stress (hardening). The yield surface determines the plastic flow in a multiaxial stress state, while a hardening law 
determines the evolution of the yield surface. The same approach is used here.

\subsection{ANISOTROPIC VEGTER YIELD FUNCTION}

The Vegter yield criterion [4] is one of the most accurate yield functions for aluminum alloy sheets defined in the principal stress space for plane stress conditions. It can easily be adapted to experimental data or physically based models. For planar anisotropic material, therefore, the yield function depends on the angle between the principal axes and the rolling direction. For a particular loading direction with respect to the rolling direction, four yield stresses are necessary to determine the model parameters: a pure shear stress, a uniaxial tensile stress, a plane strain tensile stress and an equi-biaxial stress. Between the measured stress points a Bezier curve is used to describe the yield locus. At yielding, not only the yield stress, but also the direction of plastic strain is determined. Based on Drucker's postulate, the normal to the yield locus has the same direction as the plastic strain rate. From the stress points and the strain rate directions, a set of Bezier curves can be constructed such that the resulting yield locus is continuous. In the two-dimensional principal stress space, a stress point is represented by the vector $\vec{\sigma}=\left[\sigma_{1}, \sigma_{2}\right]^{\mathrm{T}}$. Every plane stress situation can now be represented by the principal stresses $\vec{\sigma}$ and the angle $\theta$ between the 1st principal stress and the rolling direction. For every part of the yield locus between two reference stress points, $\vec{\sigma}_{i}$ and $\vec{\sigma}_{j}$, a second order Bezier function is defined. The Bezier function is determined by the two reference stress points and the direction of the yield locus at the reference points, specified by $\rho=\dot{\varepsilon}_{1} / \dot{\varepsilon}_{2}$. The intersection of the two tangents at the reference points defines the hinge point $\vec{\sigma}_{h}$ (see Figure 1). The yield locus between two reference

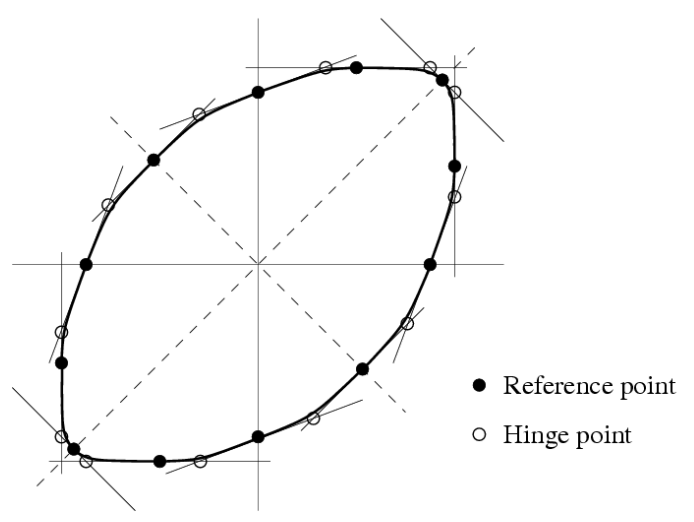

Figure 1: Basic stress points and tangents to the Vegter yield locus.

stress points is defined by

$$
\vec{\sigma}_{\mathrm{loc}}=\vec{\sigma}_{i}+2 \mu\left(\vec{\sigma}_{h}-\vec{\sigma}_{i}\right)+\mu^{2}\left(\vec{\sigma}_{i}+\vec{\sigma}_{j}-2 \vec{\sigma}_{h}\right)
$$

$\mu \in[0,1]$. A yield function is constructed by defining an equivalent stress $\sigma_{\mathrm{eq}}$ that is implied for any plane stress state by the relation

$$
\vec{\sigma}=\frac{\sigma_{\mathrm{eq}}}{\sigma_{\mathrm{f}}} \vec{\sigma}_{\mathrm{loc}}
$$

where $\sigma_{\mathrm{f}}$ is the current flow stress. A function $\phi$ that is defined as

$$
\phi\left(\boldsymbol{\sigma}, \varepsilon_{\mathrm{eq}}\right)=\sigma_{\mathrm{eq}}(\boldsymbol{\sigma})-\sigma_{\mathrm{f}}\left(\varepsilon_{\mathrm{eq}}\right)
$$

fulfils the condition that $\phi=0$ on the yield locus and $\phi<0$ in the elastic regime. The direction of the plastic strain rate can be calculated from the derivative of $\phi$ to the stress $\boldsymbol{\sigma}$. Since $\phi$ is continuously differentiable, the plastic strain rate direction is continuous. Planar anisotropic behavior can be modeled by letting all reference stress points and corresponding normals depend on the angle $\theta$. The reference stress points and normals are defined by an interpolation, based on Fourier series. A complete yield locus for one specific angle $\theta$ is presented in Figure 1, including all reference and hinge points and the tangents.

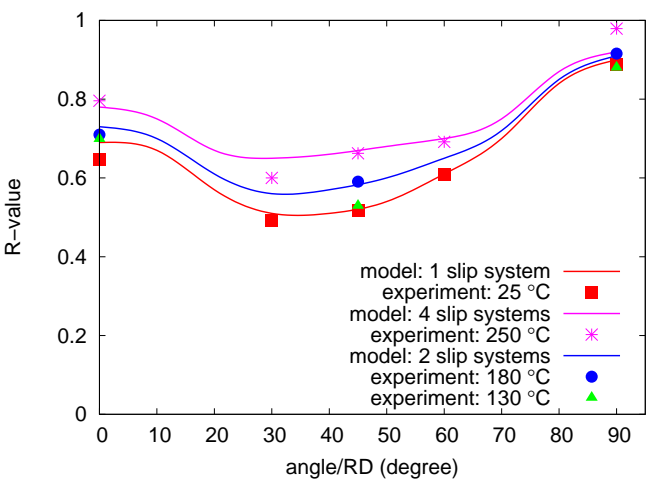

Figure 2: Lankford $R$ value measured (symbols) and calculated (lines) at different temperatures for AA 6016-T4 alloy.

For the material model to account for changes in temperature, the anisotropy coefficients which describe the state of anisotropy must be represented as a function of temperature. At room temperature, the Vegter yield surface parameters were identified from the mechanical tests proposed in [4]. The evolution of yield surface due to temperature is included by identifying the anisotropy coefficients at several temperatures from the Visco Plastic Self Consistent (VPSC) crystal plasticity model [7]. Experimental observations have shown that in addition to the octahedral slip systems other slip systems families can be activated when the temperature is increased [8]. EBSD maps measured from the deepdrawn cylindrical cups at several temperatures also revealed that at room temperature the bands are parallel to the $\{111\}\langle 110\rangle$ plane while at $250{ }^{\circ} \mathrm{C}$, these bands are also parallel to the $\{112\}$ and sometimes $\{110\},\{100\}$ planes when EBSD maps plotted in band contrast mode.

The activated slip system families and their Critical Resolved Shear Stresses (CRSS) have been obtained at specific temperatures by fitting the r-values calculated with 
the VPSC model to the r-values measured at these temperatures and for several tensile directions as shown in Figure 2. Measured r-values at $130^{\circ} \mathrm{C}$ are equal to the one at room temperature meaning that only the $\{111\}\langle 110\rangle$ slip systems are activated in this temperature range. A good fit is obtained at $180^{\circ} \mathrm{C}$ by using the $\{111\}\langle 110\rangle$ and $\{112\}\langle 110\rangle$ families with the CRSS ratio $1: 1.2$ while at $250{ }^{\circ} \mathrm{C}$ four families of slip systems $\{111\},\{110\},\{100\}$ and $\{112\}\langle 110\rangle$ are required with the CRSS ratios 0.9:1:1.1:1. For all the calculations with the VPSC model the initial experimentally measured texture has been described by 2000 orientations.

Figure 3 shows the change in the shape (stress values are normalized with respect to equivalent (uniaxial) stress, $\left.\sigma_{\text {eq }}\right)$. It is evident from Figure 3 that temperature has a distinct effect on the yield surface's shape, which must be accounted for during warm forming simulations.

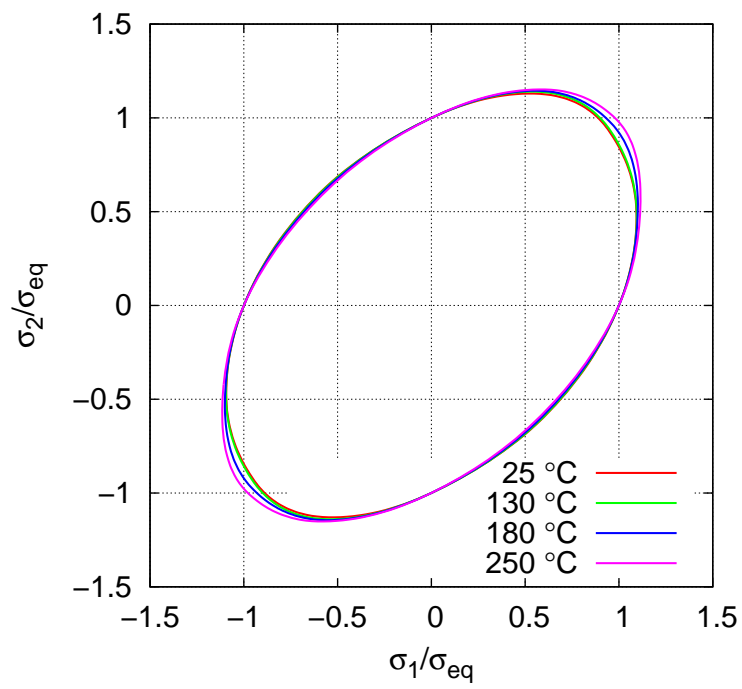

Figure 3: The Vegter yield function for AA 6016-T4 at several temperatures (stresses normalized to show the change in the yield surface shape).

\subsection{THE BERGSTRÖM MODEL}

The temperature and strain rate dependent work hardening is described by using the physically based Bergström model presented in [3]. The model considers the evolution of the dislocation density based on storage and dynamic recovery processes. More details can be found in [3].

\section{SIMULATION OF CYLINDRICAL CUP DEEP DRAWING}

In this Section, the implemented material model is discussed in terms of a case study concerned with warm deep drawing of cylindrical cups. Cylindrical cup drawing experiments were performed with a tool set of which the dimensions are given in Figure 4. Orthotropic symmetry was assumed for the material model. A quarter of the blank was modeled and boundary conditions were ap-

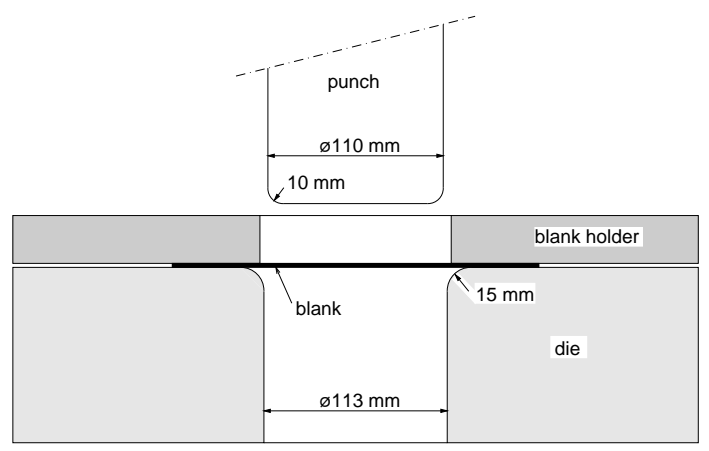

Figure 4: Dimensions of the tools for cylindrical deep drawing.

plied on the displacement degrees of freedom to represent the symmetry. The sheets were modeled with 998 discrete Kirchhoff triangular shell elements with 3 translational, 3 rotational and 1 temperature degree of freedom per node. The tools were modeled as rigid contours with a prescribed temperature. In the presented simulations the die and the blank holder were given a temperature of $250^{\circ} \mathrm{C}$, while the punch was kept at $25^{\circ} \mathrm{C}$. During deep drawing operation the area under the punch was at $25^{\circ} \mathrm{C}$ and the temperature has gradually increased from $25^{\circ} \mathrm{C}$ to $250^{\circ} \mathrm{C}$ in the wall to flange of the cup. From the VPSC calculations presented in the Section 2, the Vegter yield locus parameters were calculated only at specific temperatures, i.e. at $25,130,180$ and $250^{\circ} \mathrm{C}$. Quadratic interpolating functions were used to fit the yield locus parameters calculated at discrete temperatures.

Simulations with the temperature dependent Vegter yield locus and the Bergström hardening model implemented in the in-house implicit code DIEKA are performed at various temperatures. The global convergence criterion was set to $0.5 \%$ relative unbalance force. In the simulations a temperature dependent friction coefficient is used, with a linear relation from 0.6 to 0.12 for temperatures from $90^{\circ} \mathrm{C}$ to $110^{\circ} \mathrm{C}$ and constant before and after this range.

To investigate the influence of yield locus shape change due to temperature, warm deep drawing simulations of cylindrical cups are performed with and without considering the effect of temperature in the simulations. In Figure 5 the force-displacement diagrams of the punch, the thickness distributions of the cup at a depth of $64 \mathrm{~mm}$ and earing profiles are plotted for the experiments and the simulations. Comparing the punch force-displacement curves, it can be seen that numerical model underestimate the experimental curve in both the simulations at $250{ }^{\circ} \mathrm{C}$. This underestimation is slightly higher with the effect of temperature on yield locus. From the thickness distribution vs distance from the center of the cup curve as shown in Figure 5(b), we can observe a better agreement for the wall thickness when the effect of temperature on yield locus is included. Figure 5(c) shows that the deformation temperature strongly influences the anisotropy and earing profile. From the room temperature experiment, the earing profile exhibit four ears. When the deformation tem- 


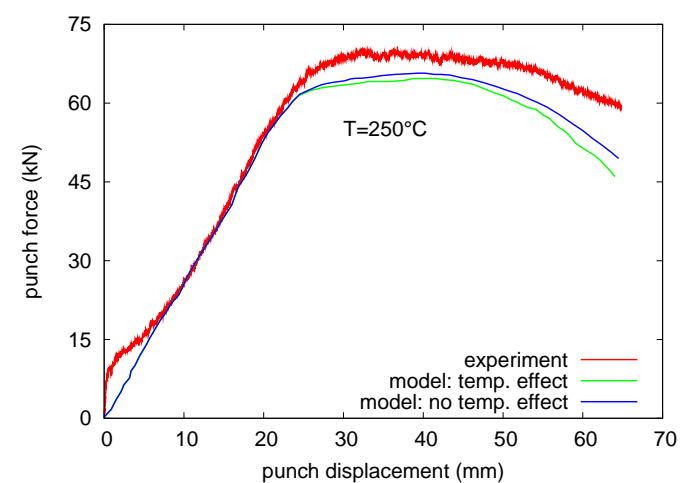

(a) Influence of temperature with yield locus on calculated punch force-displacement curves.

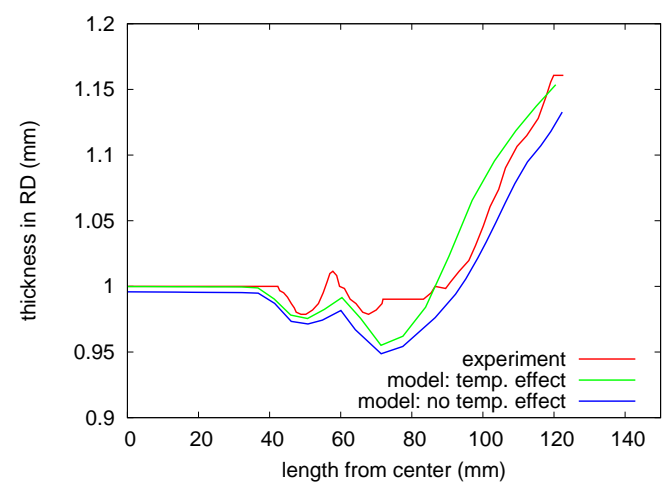

(b) Influence of temperature with yield locus on calculated thickness distribution in RD.

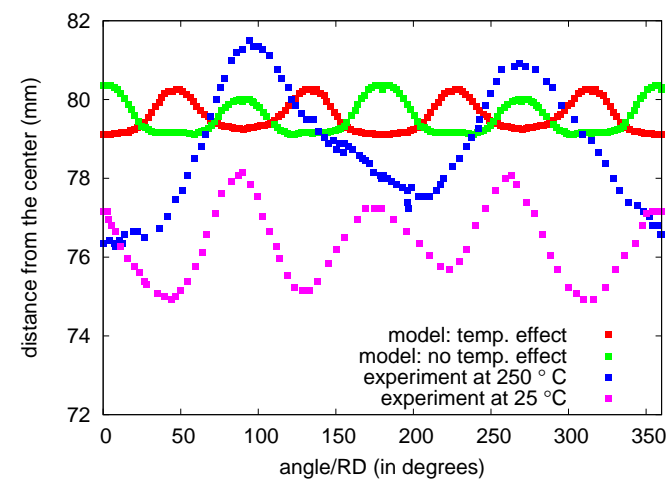

(c) Influence of temperature with yield locus on earing profiles.

Figure 5: Deep drawing of cylindrical cup-experiments and simulations (AA 6016-T4 alloy).

perature is increased to $250^{\circ} \mathrm{C}$, experimental earing profile nearly exhibit two ears. However the model cannot show the change from four to two earing profile in both situations. However, the model does show different earing profile when the effect of temperature on yield locus is included, i.e. the model including the temperature effects leads to an earing profile that is exactly opposite to the one obtained without temperature effects.

\section{CONCLUSIONS}

From the presented warm forming simulations of the cylindrical cup deep drawing, it can be concluded that the effect of temperature on shape change of yield locus has an effect notably on the predicted thickness distribution, indeed the predicted thickness with the model including temperature effects is almost overlaps the experimentally measured thickness at the bottom. In the die radius area also, the model with temperature effects performs slightly better than the one obtained without temperature effects. However the model cannot represent the experimental earing profile at elevated temperatures. The friction between tool and workpiece is one of the least known factors in the simulations. It is recommended that the friction be investigated in more detail.

\section{ACKNOWLEDGEMENT}

This research was carried out under the project number MC1.02106 in the framework of the Research Program of the Materials innovation institute M2i (www.m2i.nl), the former Netherlands Institute for Metals Research. The authors are indebted to P.J. Bolt and R. Werkhoven of TNO Science and Industry for performing the warm deep drawing experiments.

\section{REFERENCES}

[1] D. Li and A. Ghosh. Tensile deformation behavior of aluminum alloys at warm forming temperatures.

Mater. Sci. Eng. A., 352:279-286, 2003.

[2] H. Takuda, K. Mori, I. Masuda, Y. Abe and M. Matsuo. Finite element simulation of warm deep drawing of aluminum alloy sheet when accounting heat conduction. J. Mater. Proc. Tech., 120:412-418, 2002.

[3] A.H. van den Boogaard and J. Huétink. Simulation of aluminium sheet forming at elevated temperatures. Comp. Methods in Appl. Mech. and Eng., 195: 6691-6709, 2006.

[4] H. Vegter and A.H. van den Boogaard. A plane stress yield function for anisotropic sheet material by interpolation of biaxial stress states. Int. J. of Plast. 22:557-580, 2006.

[5] S. Kurukuri, A.H. van den Boogaard, A. Miroux and B. Holmedal. Warm forming simulation of Al-Mg sheet. J. Mater. Proc. Tech., Submitted.

[6] M. Merklein, W. Hußnätter and M. Geiger. Characterization of yielding behavior of sheet metal under biaxial stress condition at elevated temperatures. CIRP Annals - Manufact. Tech. 57 (1): 269-274, 2008.

[7] R.A. Lebensohn and C.N. Tomé. A self-consistent anisotropic approach for the simulation of plastic deformation and texture development of polycrystals: Application to zirconium alloys. Acta. Metall. Mater. 41 (9):2611-2624, 1993.

[8] B. Bacroix and J.J. Jonas. The influence of non-octahedral slip on texture development in FCC metals. Textures. Aniso 8 :267-311, 1988. 